In dem letzten Fall wurde die nähere Zusammensetzung der Luft nach Bunsen mit Pyrogallussäurekalium bestimmt und gefunden, dass sie aus 85,1 Vol.-Proc. Stickstoff und 14,9 Vol.-Proc. Sauerstoff bestand, dass sie also etwas reicher an Stickstoff war als die gewöhnliche Luft.

Die Wiederholung der Bestimmung der in Natronlauge unlöslichen Gase, nachdem eine bestimmte Menge Kohlensäure ausgeblasen war, ergab Folgendes:

\begin{tabular}{|c|c|c|}
\hline Flasche & Aurgeblasen & Gebalt ad fremden Gasen \\
\hline$I$ & die Hälfte & nicht mehr bestimmbar \\
\hline III & & - \\
\hline IV & Neun Zehntel & - \\
\hline V & Ein Drittel & 0,8 Vol.-Proc. \\
\hline
\end{tabular}

In Flasche II wurde diese Bestimmung nicht gemacht, da der Gehalt zu Anfang schon so gering war.

Die Menge der Kohlensäure in den Flaschen und die Rūckstände betrugen:

\begin{tabular}{|c|c|c|c|}
\hline Iasche & Inhalt & Ruckstand & Natur des Rulekstandes \\
\hline I & $10,0 \mathrm{~kg}$ & $0,5 \mathrm{~g}$ & $\begin{array}{l}\text { Wasser und Spur } \\
\text { suspendirtes Eisen- } \\
\text { oxyd. }\end{array}$ \\
\hline II & 10,3 & $520 \mathrm{~g}$ & $\begin{array}{l}517 \mathrm{~g} \text { Wasser und } \\
3 \mathrm{~g} \text { Eisenoxyd }\end{array}$ \\
\hline III & 10,1 & $1 \mathrm{~g}$ & $\begin{array}{l}\text { Wasser und Spur } \\
\text { suspendirtes Eisen- } \\
\text { oxyd }\end{array}$ \\
\hline $\begin{array}{l}\text { IV } \\
\mathrm{V}\end{array}$ & $\begin{array}{l}8,0- \\
9,9-\end{array}$ & $\begin{array}{l}\text { nichts } \\
8,5 \mathrm{~g}\end{array}$ & $\begin{array}{l}\text { Wasser und Spur } \\
\text { suspendirtes Eisen- } \\
\text { oxyd }\end{array}$ \\
\hline
\end{tabular}

Man ersieht ans diesen Analysen, dass im Allgemeinen die Reinheit der fiüssigen Koblensaure des Handels eine recht gute ist. Der Wassergehalt des erst ausgeblasenen Gases jst ganz ohne Bedeutung. Von mehr Gewicht ist der Gehalt an fremden Gasen; in dieser Beziehung war das natürliche Gas das reinste, aber auch bei den anderen war der Totalgehalt nicht gross und kann man diesen gewiss auf weniger als die Hälfte des beim Anfang gefundenen scbătzen, da, wenn die Flaschen halb leer geblasen sind, die Menge der fremden Gase schon nicht mehr bestimmbar ist. Das Vorkommen von Kohlenoxyd in dem Gase, aus Magnesit bereitet, scheint mir darauf hinzuweisen, dass die Kohlensāure nicht durch Aufschliessen mit Säure, sondern durch Erhitzung frei gemacht ist und dass das Kohlenoxyd durch reducirende Substanzen, welche dabei anwesend waren, entstanden ist. Dieses Vorkommen von Kohlenoxyd in der flüssigen Kohlensåure scheint mir doch etwas bedenklich, wenn sie in den sehr begrenzten Schiffsrãumen gebraucht werden soll, indem bei eventuellen Ondichtigkeiten der Kältemaschi- nen, welche am meisten beim Anfang des Arbeitens vorkommen kōnnen, dann gerade das sehr giftige Kohlenoxyd in der grössten Menge in die umgebende Luft entweichen wird. Ich habe denn auch die flüssige Koblensäure, welche frei ist von Kohlenoxyd, vorgezogen und unter die Lieferungsbedingungen aufuebmen lassen, dass die flüssige Kohlensäure als verunreinigende Gase nur Luft enthalten darf. Die Rückstände, welche nach dem völligen Leerblasen der Flaschen in denselben verblieben, waren nur bei der Flasche aus natürlicher Kohlensäure von Bedeutung, nämlich ungefäbr 5 Proc. von dem Gewichte der flüsoigen Kohlensăure; in den anderen Fällen erreichten sie nicht einmal 0,1 Proc. Ich kann mir keinen Grund denken, warum bei der natürlichen Kohlensăure immer nothwendiger Weise ein so grosser Gehalt an Wasser vorkommen soll, und es ist mir wabrscheinlich, dass hier, wie der Fabrikant mir versicherte, ein Verseben vorlag, indem man versãumt batte, die Flaschen, welche von den Mineralwasserfabrikanten oft mit einer gewissen Menge Wasser zurückkommen, vor der erneuten Füllung nachzusehen und das Wasser auszugiessen. Immerhin ist es ein Zeichen, dass man auch auf diese Umstände bedacht sein muss.

\section{Apparat zum Schöpfen von Wasserproben aus beliebiger Tiefe.}

Von Prof. Dr. Friedrich C. G. Müller, Brandenburg a. $\mathrm{H}$.

Der in eine Bleiplatte $A$ (Fig. 1) gelötete Bügel $B$ hängt mittels einer Spiralfeder $E$ und des Carabinerhakens $F$ an dem Ringe einer Lothleine oder eines Messbandes. Im Bügel kann eine Flasche $D$ von $400 \mathrm{ccm}$ mit Hülfe der Klemmvorrichtung $C$ befestigt werden. Die Flasche wird mit einem doppelt durchbohrten Kautschukstopfen versehen und die beiden Bobrungen mit dem U-förmigen, aus einem Glasstäbchen hergestellten Stōpsel $H$ verschlossen. Letzterer sitzt an der Kette $I$, welche durch dieSpirale bāngt und mit ibrem oberen Ende im Ringe von $F$ befestigt ist.

Es ist einleuchtend, dass, wenn diese Vorrichtung ins Wasser hinabgelassen ist, ein

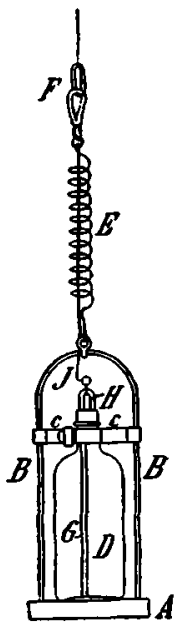

Fig. 1. lurzer Ruck an der Leine das Herauszieben des Stöpsels $H$ zur Folge bat, da ja 
die Spiralfeder sich langzieht, ohne dass die daran hängende träge Masse von etwa $1600 \mathrm{~g}$ gleich nachfolgt. Nach Entfernung des Stöpsels dringt aber das Wasser durch das in der einen Bohrung steckende Röhrchen $G$ ein, während die Luft durch die andere Bohrung entweicht. Nach spätestens 80 Secunden kann die Flasche gefüllt emporgezogen werden.

Der einfache Apparat hat sich in den Händen verschiedener Forscher als bequem, zuverlässig und zweckentsprechend erwiesen. Allerdings standen bis jetzt nur Wassertiefen bis zu $60 \mathrm{~m}$ zur Untersuchung. Man wird aber anstandslos bis auf $100 \mathrm{~m}$ hinabgehen können, ohne dass die Flasche eine gewöhnliche Medicinflasche - zerdrückt wird. Handelt es sich um grössere Tiefen, so wird die Flasche mit Benzin gefüllt und Kork nebst Stöpsel nicht ganz dicht schliessend aufgesetzt. Im Übrigen verfährt man, wie beschrieben. Das Benzin entweicht aus der Flasche nach Beseitigung des Stöpsels fast ebenso schnell wie Iuft.

Es braucht kaum gesagt zu werden, dass die angegebenen Maasse nicht verbindlich sind. Wir haben Anfangs mit einem weit leichteren Apparat gearbeitet, zu welchem die nur $100 \mathrm{ccm}$ fassenden Kölbchen des Tenax-Apparates ${ }^{1}$ ) zur Bestimmung der Wassergase passen. Mit diesem kleinen Muster arbeitet es sich ungleich bequemer, sodass man bei geringen Tiefen mit demselben vielleicht lieber viermal je $100 \mathrm{~g}$ schöpft als mit dem grösseren $400 \mathrm{~g}$ auf einmal. Über $400 \mathrm{ccm}$ hinauszugehen erscheint nicht empfehlenswerth, falls nicht maschinelle Lotheinrichtungen zur Verfügung stehen $\left.^{2}\right)$.

\section{Referate.}

\section{Physikalische Chemie.}

E. Rntherford. Eine von Thoriumverbindungen ausgesandte radioactive Substanz. (Philosophical Magazine, 5. Serie, 49, 161.)

Diese Arbeit beschäftigt sich mit der Untersuchung der unter dem Namen der radioactiven Induction bekannten Erscheinungen (vgl. H. Becquerel, Compt. rend. $1899,129,912$ und diese Zeitschrift 1900, 81: Selbststrablende Materie); während aber bisher diese secundäre Strahlung nur an Radiumpräparaten beobachtet worden war, zeigt jetzt Rutherford, dass auch Thoriumverbindungen, deren Radioactivität G. C. Schmidt nachgewiesen hatte, ibr Strahlungsvermögen auf andere Körper zu übertragen befähigt sind. Während aber die von Radiumstrahlen getroffenen Körper die empfangene Activität nur durch die gewonnene Eigenschaft - die Luft für Elektricität leitend zu machen - erkennen lassen, verhalten sich die von Thoriumverbindungen beeinflussten Körper ganz so, als wären sie selbst radioactiv: die ihnen inducirten Strahlen entladen nicht nur geladene Leiter, sondern wirken auch auf die photographische Platte ein, bieten somit die beiden Haupterkennungszeichen der Becquerelstrahlen dar. Ein Unterschied von den Primärstrahlen aber zeigt sich in ihrem Vermögen, feste Körper $z u$ durchdringen; alle von den bisher bekannten strahlungsactiven Substanzen direct ausgesandten Strahlen werden leichter von undurchsichtigen Schichten und auch von Luft absorbirt als die von Thorverbindungen erzengten Secundärstrahlen. Dass diese letzteren einen wesentlich anderen Charakter haben als die durch Radium hervorgerufenen Secundärstrablen, ist nicht anzunehmen; es wird sich vermuthlich bei näherer Prüfung der bei Radium- und Poloniumpräparaten wahrnehmbaren inducirten Strablung dasselbe Resultat ergeben, wenn nur Präparate untersucht werden, welche die gleich grosse Strahlungsintensität besitzen als Rutherford's Thoroxyd.
Die Übertragung des Strahlungsvermögens dehnt sich auf alle beliebigen - ungeladenen Gegenstände aus, welche sich in der Umgebung der Thorverbindungen befinden; sie erreichen allo denselben Grad der Strahlungsintensität, werden eine Zeit lang selbststrahlend und verlieren in 11 Stunden die Hälfte ihrer anfänglichen Activität. Befindet sich Thoriumoxyd zwischen zwei mit verschiedener Elektricität geladenen Metallplatten, so wird die Radioactivität nur auf dem negativ geladenen Leiter erzeugt. Unter Einwirkung starker elektrischer Energie kann die Radioactivität auf der Oberfläche von dünnen Drähten concentrirt werden; so konnte z. B. ein Platindraht von $1 \mathrm{~cm}$ Länge und $0,018 \mathrm{~cm}$ Dicke eine äusserst starke Strahlungsenergie an seiner Oberfläche festhalten, eine wägbare Zunahme des Drahtes war aber nicht wahrzunehmen. Theoretisch kann diese Anhänfung von Strahlungsenergie bis ins Unendliche fortgesetzt werden, da dieselbe bei genügend starker elektrischer Ladung nur von der Menge der radioactiven Substanz abhängt, welche bei dieser Übertragung ihrer Energie allmählich die Activität verliert. Der Platindraht bebält seine strahlende Kraft, wenn man ihn in kaltes oder in heisses Wasser taucht, im Gegensatz zu dem durch Radiumstrahlen influencirten Fluorcalcium, das durch Waschen mit Wasser unwirksam wurde; auch Salpetersäure bewirkt keine Verminderung der Strablung, schnell aber wird dieselbe durch Salzsäure und Schwefelsäure entfernt. Dampft man diese letzteren Säuren, nachdem der Draht mit ihnen abgespült ist, in einer Schale ein, so findet man die Schale radioactiv. Durch Abreiben mit Sandpapier ist die Activität von dem Draht ebenfalls leicht zu entfernen, ein Beweis, dass diese an die Oberfläche der Körper gebunden ist. Jedes andere Metall zeigt

1) Zeitschr, angew. Chemie 1899, 253.

2) Der beschriebene Apparat kann für $12 \mathrm{Mk}$. durch Vermittlung des Verfassers bezogen werden. 